\title{
CIUDADANOS Y PARTIDOS EN EL CONSENSO Y DISENSO SOBRE EL ESTADO DE LAS AUTONOMÍAS
}

\author{
Edurne Uriarte
}

Universidad del País Vasco

\begin{abstract}
RESUMEN
Este artículo analiza lo que se considera el problema no resuelto de la democracia española, que es el del consenso en torno a la articulación territorial del Estado. Y defiende la tesis de que la insatisfacción y el debate abiertos sobre el Estado de las Autonomías no surgen tanto de la sociedad, o de los ciudadanos insatisfechos, sino de las actitudes de los partidos políticos y de las élites. Los datos sobre las opiniones de los ciudadanos, incluso en las autonomías más "problemáticas", muestran una alta o notable satisfacción con el sistema autonómico. Y el artículo argumenta que la insatisfacción se explica fundamentalmente porque constituye para las élites autonómicas un activo electoral eficaz y un instrumento para la perpetuación del enorme poder político y social alcanzado en sus territorios. $\mathrm{Y}$ a este factor principal se suma la falta de consenso suficiente de los dos grandes partidos españoles, PP y PSOE, en torno al modelo de articulación territorial de España.
\end{abstract}

\section{INTRODUCCIÓN: EL CUESTIONAMIENTO DEL MODELO AUTONÓMICO COMO UN PROBLEMA DE LOS PARTIDOS Y DE LAS ÉLITES Y NO DE LOS CIUDADANOS}

La construcción de la democracia española tras la dictadura se ha fundamentado en dos ejes. Por un lado, es central la configuración de las instituciones democráticas en sí mismas. Pero, por otro lado, cabe hablar de un segundo eje que merece especial atención. Me refiero al Estado de las Autonomías, es 
decir, la construcción de un modelo descentralizado de Estado que diera satisfacción a los sentimientos nacionalistas y regionalistas y resolviera el problema de la articulación territorial del sistema político español. Y este segundo eje se ha tornado central en la evolución de la democracia española.

La importancia de la construcción autonómica deriva no sólo de su relevancia en el nuevo sistema político, sino también de los problemas que se han generado en su construcción. $\mathrm{Y}$, lo que es especialmente significativo, mientras que la construcción de las instituciones democráticas se ha completado exitosamente, los problemas planteados por el Estado de las Autonomías no se han conseguido resolver definitivamente aún.

Esos problemas se refieren principalmente a la insatisfacción de los nacionalismos periféricos y el consiguiente cuestionamiento permanente en torno al modelo autonómico. De hecho, uno de los debates políticos principales que se mantienen abiertos en nuestro país es precisamente el relativo al modelo de Estado. El sistema autonómico está sometido a una discusión permanente desde el inicio de la democracia, y no podemos hablar todavía hoy en día de un consenso acabado o fuerte sobre el Estado de las Autonomías. De esta forma, un balance sobre los logros de la democracia española y una valoración de su grado de éxito presentan un problema principal que es el de la no completa resolución de la articulación territorial del Estado.

Pero la existencia de este debate abierto sobre el Estado de las Autonomías no debe ocultar al mismo tiempo que el cuestionamiento del modelo autonómico, si bien tiene capacidad por sus características para afectar a todo el sistema político, ni refleja ni surge de un rechazo mayoritario de la sociedad española, sino del rechazo de los nacionalismos periféricos, y, en estrecha relación con la postura de los nacionalismos periféricos, del debate abierto entre los principales partidos políticos españoles que no han conseguido llegar a un consenso básico sobre esta cuestión.

Los tres elementos planteados en el párrafo anterior, es decir, la posición de los ciudadanos españoles, la posición de los partidos nacionalistas y la posición de los grandes partidos nacionales, son los que articulan la tesis que se pretende desarrollar en las siguientes páginas. Esta tesis sostiene básicamente que el inacabado debate sobre el Estado de las Autonomías no surge tanto de la sociedad, o de los ciudadanos insatisfechos, sino de los partidos políticos y de las élites. Fundamentalmente, de los partidos nacionalistas, que, por factores que se explicarán más adelante, mantienen abierto desde el inicio de la transición un cuestionamiento permanente del modelo de Estado. Pero a eso se suma, además, una incapacidad de los partidos nacionales para llegar a un consenso sobre un modelo acabado de Estado de las Autonomías. Y tanto en la posición de los partidos nacionalistas como en la incapacidad de consenso de los partidos nacionales, la explicación no debe buscarse en la insatisfacción de los votantes de estos partidos, sino en las posturas de las élites políticas, que, por razones muy diferentes en un caso y en otro, mantienen constante el Estado autonómico más como problema que como solución. 
El eje de la tesis planteada supone que es necesaria, por una parte, una mirada hacia los ciudadanos, o hacia la cultura política de los españoles, y, por otra parte, un análisis de las posturas de los partidos políticos, posturas que responden en buena medida a una lógica electoral independiente de los rasgos centrales de la cultura política.

El análisis del papel de los ciudadanos y de los partidos políticos en el mantenimiento del cuestionamiento del modelo de Estado es central desde la Ciencia Política y tiene un especial interés para conocer algunos de los problemas de la democracia española. Pero no debe hacernos olvidar que hay otros temas de interés desde el punto de vista politológico en relación con el Estado de las Autonomías. Destacaría dos de estas cuestiones, que, si bien no van a ser tratadas en las siguientes páginas, sí merecen especial atención. Por un lado, y en relación con las élites, hay otro aspecto de su análisis que tiene también interés en relación con la tesis planteada en estas páginas pero que no se abordará aquí. Me refiero a la creación de unas estructuras e interés de poder propios que se entrelazan con las estructuras de poder nacionales y cuya influencia en la evolución del Estado de las Autonomías es importante valorar. Por otro lado, hay un amplio campo de análisis relativo a la eficacia y los resultados de la estructura autonómica en el que la Ciencia de la Administración aún puede aportar muchos valiosos análisis necesarios para comprender el Estado de las Autonomías, no sólo desde la dinámica de poder de las élites, o desde la cultura política, o desde los problemas de legitimación, o desde la competición partidista, sino también desde la eficacia de su funcionamiento.

\section{LOS CIUDADANOS ESPAÑOLES ANTE EL ESTADO DE LAS AUTONOMÍAS}

Los numerosos datos de encuesta relativos a la relación de los ciudadanos españoles con el Estado de las Autonomías muestran fundamentalmente una alta satisfacción con la construcción autonómica. Básicamente, la relación de los ciudadanos españoles con las autonomías, con su carácter y sus resultados, es positiva. $\mathrm{Y}$ en este sentido cabe plantear la tesis de que los problemas de cuestionamiento del Estado de las Autonomías no surgen de un estado generalizado de insatisfacción ciudadana. Es más, como veremos más adelante, tampoco cabe defender ese estado de insatisfacción para los ciudadanos de las Comunidades Autónomas donde el Estado de las Autonomías tiene más problemas de deslegitimación.

Cabe entender esta satisfacción en el contexto de la visión positiva que los españoles tienen sobre la construcción de la democracia en general, sobre la transición democrática y sobre la Constitución. En ese sentido, quizá habría que pensar que no se trata sobre todo de una satisfacción con el sistema autonómico, sino de una satisfacción global con el sistema político que alberga a ese sistema autonómico. 
En este contexto, son necesarias investigaciones que profundicen más en las relaciones de los españoles con las autonomías, con el concepto de Estado centralizado o con las identidades asociadas a las autonomías que nos puedan dar un cuadro más completo del significado de las autonomías entre los valores de los españoles.

Al margen de lo que puede dar de sí esa mayor profundización sobre los valores relativos a las autonomías, los datos de encuesta muestran que las instituciones autonómicas han sido integradas por los españoles como una estructura más de las instituciones de la democracia española, y, en ese sentido, igual de importante o necesaria que otras instituciones.

Como señalaba más arriba, es importante tener en cuenta que la visión positiva sobre el Estado de las Autonomías de los españoles se enmarca en una concepción también muy positiva de la transición y de las instituciones democráticas surgidas de esa transición. En una encuesta realizada con motivo del 20 aniversario de la Constitución, en 1998, y a la pregunta sobre la percepción en torno a la forma en que se llevó a cabo la transición, nada menos que un 79,8 por ciento de los encuestados respondía que la forma en que se llevó a cabo la transición a la democracia constituye un motivo de orgullo para los españoles ${ }^{1}$.

En diciembre de 2000, y con motivo del vigésimo quinto aniversario de la muerte de Franco, el Centro de Investigaciones Sociológicas realizaba otra encuesta en torno a las percepciones de los españoles sobre los cambios producidos en estos años. Dicha encuesta mostraba, en primer lugar, que entre los españoles había una acusada percepción sobre la importancia del cambio producido, dado que casi un 54 por ciento pensaba que la sociedad española había cambiado mucho, a lo que se añadía otro 40 por ciento que pensaba que había cambiado bastante. Pero, además, un 29 por ciento señalaba que el cambio había sido positivo y otro 57 por ciento pensaba que había sido bastante positivo. Y en la misma encuesta, por otra parte, el porcentaje de los que pensaban que la transición a la democracia constituye un motivo de orgullo para los españoles había pasado del 79,8 por ciento de 1998 al 86,1 por ciento ${ }^{2}$.

En el contexto anterior cabe entender que también haya una percepción mayoritariamente positiva sobre las Comunidades Autónomas. Cuando en 2002 se preguntaba a los españoles si pensaban que, en general, la creación y desarrollo de las Comunidades Autónomas ha sido algo más bien positivo o más bien negativo, un 67,4 por ciento respondía que ha sido algo más bien positivo. Pero, además, a lo largo de estos años y al hilo de la construcción autonómica, también se ha desarrollado entre los españoles la percepción de la importancia de las instituciones autonómicas y su influencia en la vida de los ciudadanos. Todo tipo de cuestiones que comparan los efectos de las decisiones del Gobierno central o del Gobierno autónomo muestran invariablemente que los ciudadanos equiparan la influencia de las decisiones

CIS, Estudio n. ${ }^{\circ}$ 2309, Constitución e instituciones. 20 Aniversario de la Constitución.

${ }^{2}$ CIS, Estudio n. ${ }^{\circ}$ 2401, 25 años después, diciembre 2000. 


\section{TABLA 1}

\section{Influencia de las decisiones del Gobierno central, del Gobierno autónomo $y$ del Ayuntamiento en el bienestar de los ciudadanos, 2002}

\begin{tabular}{|c|c|c|c|}
\hline & Gobierno central & Gobierno autónomo & Ayuntamiento \\
\hline Mucho ..................... & 24,3 & 23,9 & 27,3 \\
\hline Bastante .................... & 47,4 & 46,3 & 44,1 \\
\hline Poco ............................ & 17,2 & 18,3 & 17,6 \\
\hline 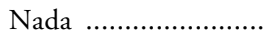 & 5,5 & 5,5 & 5,8 \\
\hline 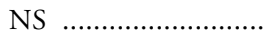 & 5,3 & 5,5 & 4,8 \\
\hline NC $\ldots \ldots \ldots \ldots \ldots \ldots \ldots \ldots$ & 0,3 & 0,4 & 0,4 \\
\hline
\end{tabular}

FUENTE: CIS, Estudio n.o 2455, Instituciones y autonomías II, septiembre 2002.

de ambos. Tal y como podemos observar en la tabla 1, la percepción sobre la influencia de las decisiones del Gobierno autónomo en el bienestar de los ciudadanos es semejante a la que se tiene sobre la influencia del Gobierno central.

En el contexto de todos esos datos positivos sobre la relación de los españoles con las autonomías, cabe preguntarse cuáles son los problemas o las raíces de los problemas de deslegitimación del Estado de las Autonomías. $\mathrm{Y}$ es ahí donde debemos centrarnos en las cuestiones relativas a la identidad, por un lado, y al modelo de Estado, por otro. Ambas están en parte conectadas, aunque no totalmente. Y en el análisis de ambas cuestiones cabe desarrollar, por un lado, una mirada nacional y, por otro lado, una mirada específica sobre los ciudadanos de las autonomías donde más problemas se plantean.

La mirada nacional sobre esta cuestión es relevante para situar el problema de la deslegitimación del Estado autonómico en sus auténticas dimensiones, y, específicamente, para llamar la atención sobre la capacidad sobredimensionada que los nacionalismos étnicos tienen para determinar el debate autonómico. En buena medida, ese problema de deslegitimación parte de la percepción sobre la propia identidad y el reflejo de esa identidad en una concepción de la propia Comunidad Autónoma como una nación o como una región. La disyuntiva entre la región y la nación es, al fin y al cabo, el eje sobre el que se sustentan los debates básicos sobre el Estado de las Autonomías.

Una mirada global sobre las percepciones de los españoles en torno a esta cuestión muestra que la percepción de la propia Comunidad Autónoma como una nación es notablemente débil y que es preciso tener en cuenta ese dato a la hora de valorar los sentimientos de identidad. Tal y como vemos en la tabla 2 , sólo un 12,3 por ciento de los españoles percibe a su Comunidad Autónoma como una nación, y la gran mayoría la identifica como una región. 


\section{TABLA 2}

Términos preferidos para referirse a la propia Comunidad Autónoma, 2002

\begin{tabular}{|c|c|}
\hline Términos & Porcentaje \\
\hline Una región .................... & 75,9 \\
\hline Una nación & 12,3 \\
\hline Ninguno de los dos, otros términos .................. & 7,0 \\
\hline NC & 4,9 \\
\hline
\end{tabular}

FUENTE: CIS, Estudio n. ${ }^{\circ}$ 2455, Instituciones y autonomías II, septiembre 2002.

Debemos entender los sentimientos de identidad de los españoles en el contexto de esta primacía de la idea de región y la compatibilidad, por lo tanto, de las Comunidades Autónomas con la idea de nación española o España como entidad que las agrupa e integra. Estos sentimientos de identidad, tal como observamos en la tabla 3, son mayoritariamente compartidos, y la identidad autonómica se conjuga con la identidad española.

TABLA 3

Los sentimientos de identidad de los españoles, 2002

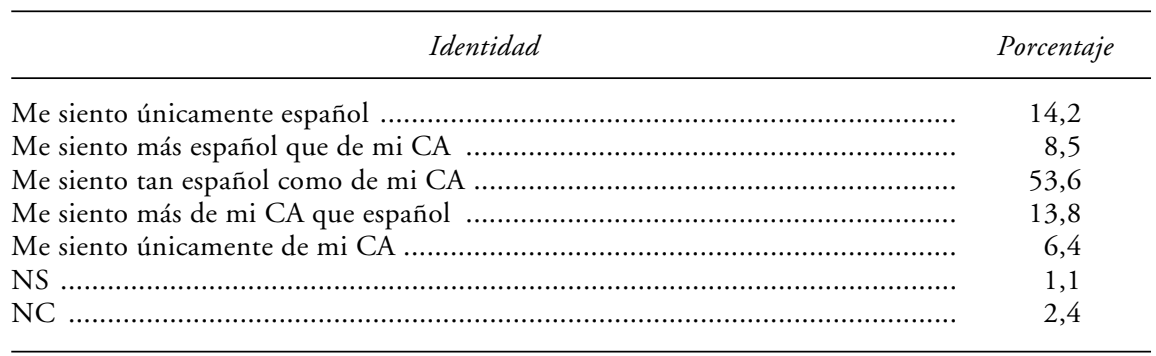

FUENTE: CIS, Estudio n. ${ }^{\circ}$ 2455, Instituciones y autonomías II, septiembre 2002.

Los datos anteriores nos muestran, además, que el cuestionamiento del modelo autonómico no es de fondo en la mayoría de los españoles, y que, por lo tanto, el debate sobre las Comunidades Autónomas es fundamentalmente un debate sobre su eficacia o sobre el número de competencias, y no tanto un debate sobre las bases fundamentales del sistema. Porque, tal como vemos en la tabla 4, tan sólo un 7,5 por ciento de los españoles defiende para su Comunidad Autónoma la posibilidad de un derecho de autodeterminación que permitiera la independencia de las Comunidades Autónomas que lo desearan. Y la 
TABLA 4

Modelos de organización territorial, 1998

Modelos de organización territorial

Porcentaje

Que dejara de ser una Comunidad Autónoma y formara parte de un Estado con un único Gobierno central

11,3

44,5

29,1

6,5

1,1

Fuente: CIS, Estudio n.o 2309, Constitución e Instituciones. 20 Aniversario de la Constitución, diciembre 1998.

gran mayoría de ese 29,1 por ciento que opta por la categoría de que «siguiera siendo una Comunidad Autónoma, pero aumentando sus competencias» no plantea un cuestionamiento del modelo autonómico, sino un simple fortalecimiento de las atribuciones de su propia autonomía.

Ciertamente, el cuestionamiento de la organización territorial del Estado es mucho más intenso en algunas Comunidades Autónomas específicas, de la misma forma que los sentimientos de identidad son más complejos o problemáticos en relación con el modelo de Estado. Pero esto no debe hacernos perder de vista esa visión global de lo que ocurre con el conjunto de los españoles en esta materia. Y esta reflexión es importante en la medida en que el debate sobre el modelo de Estado está excesivamente condicionado por unos determinados partidos nacionalistas y pierde de vista en demasiadas ocasiones los datos globales sobre el conjunto de la sociedad española.

Existe, por otra parte, otro dato relativo a las percepciones generales de los españoles en torno al modelo de Estado que tiene cierto interés y que también se tiende a olvidar. Me refiero a la percepción sobre las desigualdades regionales, y, más específicamente, a los privilegios de unas regiones sobre otras. La experiencia de los años de democracia hace pensar que las percepciones sobre la discriminación no están teniendo efectos relevantes sobre el consenso en torno al modelo autonómico, pero es importante no olvidar estas percepciones, entre otras cosas porque se les ha prestado muy poca atención desde el análisis politológico. Datos del CIS de 1996 muestran que hay un porcentaje significativo de españoles, un 30 por ciento, para quienes las diferencias de prosperidad o riqueza entre las distintas Comunidades Autónomas han tendido a aumentar.

Ciertamente, la opinión anterior está relacionada en buena medida con 
datos económicos independientes de la estructura autonómica. Porque hay una serie de desigualdades económicas entre las regiones españolas relacionadas con variables no políticas. Pero lo interesante de esta cuestión para el debate autonómico es que se acompaña de una percepción agudizada de un trato desigual a las Comunidades Autónomas por parte del Gobierno central. Porque en la misma encuesta citada más arriba, nada menos que un 71 por ciento de españoles respondía que el Gobierno central favorece más a unas Comunidades que a otras, frente a tan sólo un 16 por ciento que pensaba que el trato es igual a todas. Y a esto se añadía, además, una percepción muy pronunciada de que la Comunidad Autónoma más favorecida es, con gran distancia sobre las demás, Cataluña, seguida por Andalucía y País Vasco ${ }^{3}$.

A pesar de los datos anteriores, no cabe concluir que la percepción del trato desigual a unas Comunidades sobre otras tenga especial relevancia en el debate autonómico actual, en buena medida porque ese debate gira fundamentalmente sobre otras cuestiones ya apuntadas en la introducción y que se abordarán a continuación. Otro problema es que esta percepción de las diferencias pueda tener alguna relevancia en el futuro o pueda estar desarrollando algunos sentimientos negativos sobre la estructura autonómica o las actitudes de algunos partidos nacionalistas que acaben teniendo alguna influencia en las relaciones de los españoles con las instituciones autonómicas.

\section{EL DISENSO SOBRE EL MODELO AUTONÓMICO Y LAS ÉLITES NACIONALISTAS}

En un interesante trabajo sobre la transición española, Richard Gunther argumentaba que la exitosa transición democrática se debió fundamentalmente a una profunda transformación de las élites políticas españolas desde la desunión a la unidad consensual. Gunther señalaba que la transformación implicó procesos de acuerdos entre élites que se reflejaron en las intensas rondas de negociaciones que tuvieron lugar entre la segunda mitad de 1976 y el final de 1978, y que culminaron con la abrumadora aprobación de la Constitución en el referéndum de diciembre de $1978^{4}$.

Parece indudable que la disposición al acuerdo entre las élites, las que provenían del régimen franquista y las que se opusieron a él, es fundamental para explicar el éxito de la transición política española. También para entender y explicar la transformación de la articulación territorial de nuestro país. Porque también en relación con esta cuestión se desarrollaron procesos de negociación entre las élites que llegaron al consenso sobre los ejes básicos de la descentrali-

CIS, Estudio 2211, Datos de Opinión 5. Diferencias regionales, marzo 1996.

4 Richard Gunther, «Spain: the very model of the modern elite settlement», en John Higley y Richard Gunther (eds.), Elites and Democratic Consolidation in Latin America and Southern Europe, Cambridge University Press, Cambridge, 1992, p. 39. 
zación. El problema de ese consenso, sin embargo, era doble. Muy especialmente, debido a un factor cuya capacidad desestabilizadora ha permanecido hasta nuestros días. Me refiero a la falta de disposición de las élites nacionalistas para llegar a ese consenso. Y a ello se unía un segundo elemento también importante. $\mathrm{Y}$ es que el resto de las élites políticas nacionales arrastraron hasta el presente algunas interrogantes sobre el modelo de articulación territorial que han impedido fortalecer un consenso sólido sobre el Estado de las Autonomías, consenso con capacidad de respuesta a las constantes exigencias nacionalistas de nuevos cambios o nuevos modelos.

En el inicio de la transición, la necesidad de dar una respuesta al problema de la articulación territorial del Estado era fundamental. El franquismo había desarrollado un modelo centralista que agravó aún más la cuestión regional, que era, por otra parte, anterior al franquismo. España presentaba unos sentimientos de identidad propios en algunas regiones que requerían un nuevo modelo político que diera respuesta a esos sentimientos y que continuara un proceso que se había iniciado en la II República, con la aprobación del Estatuto catalán en 1932 y del Estatuto vasco en 1936.

La diferencia entre la II República y la actualidad es que entonces tan sólo había unas pocas regiones autónomas y no un sistema generalizado de autonomías, y en este punto algunos autores se han preguntado sobre lo que hubiera ocurrido si no hubiera llegado la Guerra Civil y la República hubiera generalizado el sistema de autonomías, una pregunta que tiene interés en relación con el debate que se dio en la transición, y que en cierta forma se ha prolongado hasta el presente, en torno a la generalización del sistema de autonomías a todas las regiones españolas.

La Constitución de 1978 consagra el nuevo Estado de las Autonomías en su artículo 2, que reconoce la autonomía de las nacionalidades y regiones de España, si bien ya antes de la aprobación de la Constitución se estableció un sistema de pre-autonomías. Se formaron asambleas de parlamentarios en todas las regiones con los diputados y senadores elegidos en las elecciones generales que consensuaron una pre-autonomía y constituyeron los primeros órganos de poder de las futuras autonomías.

A pesar de que había bastantes diferencias respecto al grado y tipo de descentralización deseables entre los partidos que consensuaron la Constitución, el rasgo más importante de sus posturas era la voluntad de dar una solución política a las identidades nacionales y regionales de España. Y esta voluntad no sólo se expresó en la articulación de la descentralización, sino también en la introducción del concepto de «nacionalidades». De hecho, el artículo segundo de la Constitución señala que «la Constitución se fundamenta en la indisoluble unidad de la nación española, patria común e indivisible de todos los españoles, y reconoce y garantiza el derecho a la autonomía de las nacionalidades y regiones que la integran y la solidaridad entre ellas».

El uso del término de «nacionalidades» junto al de regiones, y todo ello al lado del concepto de "nación española», reflejaba y refleja, por un lado, la 
complejidad de la cuestión regional-nacional en España y, por otro lado, las dificultades de dar una respuesta plenamente satisfactoria para todas las sensibilidades. De hecho, el término de "nacionalidades» suscitó debates en su momento por las dificultades de compatibilidad que este término planteaba respecto al concepto de nación española.

En un libro publicado en 1982, Manuel Fraga escribía que Alianza Popular había propuesto el artículo 5, equivalente al artículo 2, en el que se señalaba: «El Estado español, uno e indivisible, reconoce y fomenta las autonomías regionales, provinciales y locales; practica en sus propios servicios la más amplia desconcentración, y tendrá en cuenta en toda su legislación los principios de autonomía y descentralización». Porque el problema, para Manuel Fraga, era la introducción del concepto de nacionalidades dado que, nos dice, "se iniciaba así un camino peligroso: primero, de confusión terminológica, pues en el artículo 1..$^{\circ}$ se habla del "pueblo español" como fuente de todos los poderes del Estado, y aquí surge una pluralidad de pueblos; segundo, de introducción de un término de riesgos ("principio de nacionalidades", "autodeterminación", etc.), y tercero, de discriminación ("nacionalidades" de primera y "regiones" de segunda)»".

Y, ciertamente, las dificultades para compatibilizar esas nacionalidades con la nación española, o los problemas de diferenciación entre nacionalidades de "primera categoría» o regiones de «segunda categoría», se han prolongado hasta el presente. Pero lo cierto es que en algunas regiones españolas había unos sentimientos de identidad propia que necesitaban de algún tipo de plasmación en la Constitución, y por eso la redacción de la Constitución era inevitable y necesaria. Y el problema no estaba tanto en los conceptos recogidos, sino en la actitud futura de algunos nacionalismos ante una Constitución cuya inspiración y objetivo era la convivencia de distintos sentimientos de región o de nación en el seno de la nación española que agrupaba a todos.

De hecho, y en lo que se refiere al modelo de articulación territorial, la Constitución de 1978 pretende responder sobre todo a las aspiraciones de los nacionalistas vascos y catalanes. Pero, en la práctica, el sistema autonómico generaliza la fórmula autonómica a todas las regiones españolas y ofrece las mismas posibilidades de descentralización y de afirmación de la identidad regional o nacional a todas las regiones.

Y este proceso de generalización de la autonomía para todos se produce de dos formas. Inicialmente, y como ha señalado Eliseo Aja, se deducía de la Constitución que habría dos grupos de Comunidades Autónomas con un nivel distinto de competencias, un nivel más alto para las Comunidades con mayores aspiraciones históricas de autonomía y uno más bajo para el resto de regiones. Pero, como también añade Aja, este diferente nivel no se estableció de forma cerrada, sino que la Constitución tan sólo indicaba procedimientos y

Manuel Fraga Iribarne, «La Constitución española de 1978: su elaboración, la actitud de los partidos políticos y la experiencia tras un año largo de vigencia», en VV.AA., España 19751980. Conflictos y logros de la democracia, José Porrúa Turanzas Ediciones, Madrid, 1982, p. 146. 
dejaba un campo abierto para que estos procedimientos pudieran seguir caminos que no estaban inicialmente fijados ${ }^{6}$.

En la práctica, la evolución posterior, desde la aprobación de los Estatutos de Autonomía hasta la actualidad, ha orientado la articulación de las autonomías hacia una creciente equiparación de todas ellas en sus competencias y ha roto la posible línea inicial de los dos niveles de autonomía. Porque tanto en sus competencias como en la percepción que tienen de sí mismas en la actualidad las regiones españolas, lo cierto es que se han equiparado en poder y en deseos de mantener ese poder.

La equiparación de las autonomías no estaba clara en sus inicios, debido fundamentalmente a los muy diferentes sentimientos de singularidad que existían en las regiones españolas. Pero el resultado final de la construcción del Estado de las Autonomías no es sorprendente a la luz de dos procesos lógicos e inevitables. En primer lugar, los deseos de igualdad de las regiones o los agravios comparativos ante la posibilidad de desarrollo de autonomías de "primera" o «segunda» categoría. En segundo lugar, la creación y desarrollo de unas élites políticas alrededor de las nuevas instituciones autonómicas y una red de intereses de todo tipo alrededor de esas instituciones que, una vez puestas en marcha, tendían a perpetuarse.

Aunque el segundo elemento se ha hecho crecientemente importante, sólo el primero es suficiente para explicar que no era posible hacer un sistema autonómico desigual, con «naciones» con una autonomía fuerte y «regiones» con una autonomía débil. Ese modelo hubiera dado lugar a unas percepciones de desigualdad insoportables, como ya parece claro cuando miramos este proceso en retrospectiva.

Pero el problema de la construcción autonómica no es tanto el de la generalización del modelo a todas las regiones, a pesar de que se ha barajado en más de una ocasión la hipótesis de cuál hubiera sido el resultado de un sistema autonómico con niveles de autonomía sustancialmente diferentes entre las nacionalidades y las regiones. El problema de la construcción autonómica es el de la insatisfacción de los nacionalismos periféricos, es decir, de las élites nacionalistas, con esa construcción autonómica. A pesar de que la autonomía se construye con la mirada puesta en los nacionalismos, lo cierto es que la insatisfacción fundamental proviene de esos nacionalismos.

En este punto cabe hacer una diferenciación entre el nacionalismo catalán y el nacionalismo vasco, puesto que es indudable que los problemas de deslegitimación del modelo autonómico provienen fundamentalmente del nacionalismo vasco. Y no sólo por la existencia y persistencia de un terrorismo de ideología ultranacionalista, sino también por las actitudes de unas élites nacionalistas vascas cuestionadoras de los consensos.

Juan Pablo Fusi ha establecido en su último libro una pertinente diferenciación entre las actitudes del nacionalismo vasco y catalán. Fusi ha destacado

${ }^{6}$ Eliseo Aja, op. cit., pp. 55 y 56. 
que la Constitución de 1978 fue aprobada en Cataluña con el 61,6 por ciento de votos del censo, y que Jordi Pujol ha afirmado muchas veces que Cataluña asumía plenamente la unidad del Estado español. Fusi añade, además, otro dato muy importante, que es el del apoyo de CiU tanto al Gobierno de Felipe González de 1993-1996 como al de José María Aznar de 1996-2000. Otra cuestión es si de estos datos cabe hacer una afirmación tan positiva como la que hace Juan Pablo Fusi de que «el nacionalismo catalán (...) actuó, por ejemplo, a todo lo largo de la transición española y aún después, en el periodo 1975-2000, como una fuerza de estabilización y equilibrio» ${ }^{7}$.

Pero lo que es indudable es la importancia de la diferenciación entre el nacionalismo catalán y el vasco, que Juan Pablo Fusi destaca con datos como la oposición del PNV a la Constitución porque no recogía otra fuente de soberanía que no fuera la española, o el hecho de que la Constitución fuera aprobada en el País Vasco con tan sólo el 42,3 por ciento del censo electoral ${ }^{8}$. Y a todo eso habría que añadir, lógicamente, toda la evolución posterior del nacionalismo, con un paulatino acercamiento del nacionalismo moderado al nacionalismo radical que se plasmó, en primer término, en el Pacto de Lizarra de 1998 y, recientemente, en el llamado Plan Ibarretxe.

La evolución del nacionalismo vasco se ha dirigido a una abierta defensa del llamado derecho de autodeterminación, un elemento que se ha hecho explícito en el discurso del nacionalismo moderado en estos últimos años. $\mathrm{Y}$ esto ha significado que la insatisfacción con el modelo autonómico, que durante años se reflejó únicamente en una exigencia de una autonomía más profunda, de mayores competencias y de más transferencias, se ha tornado en los últimos años en un apoyo a las posiciones independentistas. El reflejo más importante de la nueva defensa abierta de las posiciones independentistas y que reúne por igual al Partido Nacionalista Vasco y a Eusko Alkartasuna es el llamado Plan Ibarretxe, presentado en otoño de 2002, que defiende el llamado Estado libre asociado. El elemento fundamental de este modelo es que los nacionalistas reclaman la soberanía para el País Vasco, soberanía para tener la capacidad de decidir una posible independencia. El Plan Ibarretxe, además, defiende la celebración de un referéndum sobre la cuestión que se celebraría tras un acuerdo en el Parlamento vasco, e independientemente de que exista o no un acuerdo de las Cortes Generales.

La radicalización del nacionalismo vasco es de enormes dimensiones porque no sólo defiende abiertamente la independencia, sino que, además, plantea una ruptura abierta con el Estado a través de un referéndum que los nacionalistas afirman van a convocar sin la aquiescencia de las instituciones del Estado. El claro y abierto independentismo de los nacionalistas vascos es un elemento sustancialmente nuevo de su discurso, puesto que hasta recientemente uno de los dos rasgos fundamentales que diferenciaban al nacionalismo mode-

Juan Pablo Fusi, La patria lejana, Taurus, Madrid, 2003, p. 303.

${ }^{8}$ Juan Pablo Fusi, ob. cit. 
rado del nacionalismo radical de Batasuna era precisamente que el radical defendía la independencia, mientras que el moderado era autonomista. Pero esta línea de diferenciación ha desaparecido y, en términos de objetivos políticos, el nacionalismo vasco se ha unificado.

Este proceso de radicalización ha diferenciado aún más al nacionalismo vasco del catalán, puesto que el nacionalismo catalán ha mantenido a lo largo de estos años un proceso de diálogo permanente con el Gobierno central y, si bien con ambigüedades, ha tendido a aceptar los consensos fundamentales de nuestro sistema político. Eso sí, siempre ha mantenido una puerta abierta a nuevas reivindicaciones, una exigencia de mayor autonomía, exigencia que en los últimos tiempos se ha traducido en la petición de un nuevo Estatuto, y, además, nunca ha dejado a un lado la posibilidad de reivindicar el derecho de autodeterminación y la independencia, algo a lo que el nacionalismo catalán no renuncia, aunque no muestre un interés inmediato.

A los nacionalismos vasco y catalán se une el nacionalismo gallego, de interés menor por su mucho más débil peso electoral y, por lo tanto, por su menor capacidad para influir en el devenir del sistema político y, en este caso, en su cuestionamiento y deslegitimación. A pesar de esa menor fuerza, es indudable que el BNG juega un papel relevante en la política gallega y que su posición de cuestionamiento del sistema autonómico es también significativa en el debate sobre el Estado de las Autonomías.

A pesar de las importantes diferencias entre estos nacionalismos, y, muy especialmente, a pesar de que el único nacionalismo que mantiene una posición de oposición frontal a los consensos fundamentales sobre la articulación territorial del Estado es el nacionalismo vasco, todos ellos han contribuido durante estos años al disenso permanente sobre el Estado de las Autonomías. El nuevo sistema político español que se construyó tras la dictadura ha presentado desde sus inicios un importante problema de cuestionamiento de uno de sus ejes fundamentales, el de la respuesta que se le dio a la cuestión de la articulación territorial del Estado.

Y la tesis que se defiende en estas páginas es que ese cuestionamiento no responde tanto a una demanda ciudadana o a una insatisfacción ciudadana importante que requiriera una revisión del modelo autonómico, sino a las actitudes de unas élites políticas nacionalistas que han hecho de la confrontación con el Estado un activo electoral bastante eficaz y, también, un instrumento para la perpetuación y permanencia del enorme poder político y social alcanzado en sus ámbitos territoriales.

Si nos atenemos, en primer lugar, a los datos sobre la evolución de la identidad en España, el aspecto más interesante de esa evolución es que tanto en los primeros años de la democracia como en la actualidad las identidades son en España fundamentalmente compartidas. Es decir, los ciudadanos españoles integran sin problemas la identidad española con la identidad correspondiente a cada Comunidad Autónoma. Y los datos de la tabla 5, elaborada por Ramón Máiz, Pablo Beramendi y Mireia Grau, muestran precisamente 
que esto es así y que la implantación y desarrollo del sistema autonómico no ha cambiado el rasgo más importante de las percepciones de identidad en España, es decir, la percepción mayoritaria de una identidad fundamentalmente dual o compartida.

No sólo eso. Además, los datos de la tabla 5 muestran que no ha habido un fortalecimiento de la identidad exclusivamente de la Comunidad Autónoma, sino más bien un debilitamiento. Y esto ocurre no sólo en el conjunto de las Comunidades Autónomas contenidas en la tabla 5, sino también, específica-

\section{TABLA 5}

La evolución de las identidades

\begin{tabular}{|c|c|c|c|}
\hline & \multicolumn{3}{|c|}{1979} \\
\hline & $\begin{array}{l}E x \\
\text { Esp. }\end{array}$ & Dual & $\begin{array}{l}E x . \\
C A\end{array}$ \\
\hline Cataluña ...... & 33,97 & 52,86 & 13,16 \\
\hline Galicia .......... & n.a. & n.a. & n.a \\
\hline País Vasco.... & 19,75 & 53,25 & 26,98 \\
\hline Canarias ....... & 25,20 & 57,74 & 14,38 \\
\hline Andalucía .... & n.a. & n.a. & n.a. \\
\hline Aragón ......... & n.a. & n.a. & n.a. \\
\hline C. Valenciana & 46,42 & 48 & 0,033 \\
\hline Madrid ........ & n.a. & n.a. & n.a. \\
\hline La Rioja ....... & n.a. & n.a. & n.a. \\
\hline
\end{tabular}

\begin{tabular}{|c|c|c|c|c|c|c|c|c|c|c|}
\hline & \multicolumn{5}{|c|}{$1985-1986$} & \multicolumn{5}{|c|}{1996} \\
\hline & $\begin{array}{l}E x \\
\text { Esp. }\end{array}$ & $\begin{array}{l}\text { Más } \\
\text { Esp. }\end{array}$ & Igual & $\begin{array}{c}\text { Más } \\
C A\end{array}$ & $\begin{array}{l}E x \\
C A\end{array}$ & $\begin{array}{l}E x \\
\text { Esp. }\end{array}$ & $\begin{array}{l}\text { Más } \\
\text { Esp. }\end{array}$ & Igual & $\begin{array}{c}\text { Más } \\
C A\end{array}$ & $\begin{array}{l}E x \\
C A\end{array}$ \\
\hline Cataluña ..... & 11 & 19 & 48 & 8 & 11 & 11,9 & 11,5 & 36,5 & 25,7 & 11 \\
\hline Galicia ......... & 5 & 7 & 52 & 27 & 6 & 4,8 & 7,8 & 43,7 & 35,7 & 7 \\
\hline País Vasco ... & 10 & 4 & 36 & 28 & 28 & 5,3 & 4 & 36,3 & 29,8 & 20,7 \\
\hline Canarias ...... & 13,6 & 2,1 & 37,1 & 16,4 & 27,9 & 5,5 & 2,7 & 45,6 & 33,6 & 10,9 \\
\hline Andalucía .... & 7 & 7 & 63 & 18 & 2 & 5 & 10,1 & 67,9 & 12,6 & 3,2 \\
\hline Aragón ......... & 13 & 5 & 66 & 11 & 2 & 8,9 & 10,1 & 63,3 & 17,7 & 0 \\
\hline C. Valenciana & 17 & 18 & 53 & 9 & 1 & 19,4 & 14,8 & 55,5 & 9,8 & 1,3 \\
\hline Madrid ........ & n.a. & n.a. & n.a. & n.a. & n.a. & 31,1 & 20,4 & 44 & 2,2 & 0 \\
\hline La Rioja ....... & n.a. & n.a & n.a. & n.a. & n.a. & 0 & 5,9 & 82,4 & 11,8 & 0 \\
\hline
\end{tabular}

Fuente: Ramón Máiz, Pablo Beramendi y Mireia Grau, «La federalización del Estado de las Autonomías: evolución y déficit institucionales», en Joan Subirats y Raquel Gallego (eds.), Veinte años de autonomías en España, CIS, Madrid, 2002, p. 411. 
mente, en las Comunidades con nacionalismos más fuertes. No se ha producido, en este sentido, una relación crecientemente problemática de los ciudadanos con la identidad española que sugiriera un fondo de problemas importantes de relaciones con el sistema político en su conjunto, y, fundamentalmente, con la solución dada por ese sistema a la plasmación política de las identidades diferenciadas.

Es cierto que tanto en Cataluña como en Galicia se ha producido un importante aumento de las identidades definidas como más de su propia Comunidad Autónoma. Pero también aquí cabe destacar sobre todo que la identidad mayoritaria, también la que se define como más propia de la Comunidad Autónoma, sigue siendo compartida. Y, además, y esto es importante, las variaciones que se producen en la Comunidad Autónoma que contiene el nacionalismo menos tendente al consenso, es decir, en el País Vasco, son variaciones de disminución de la identidad exclusiva.

En definitiva, el desarrollo del Estado de las Autonomías no ha dado lugar a unos ciudadanos crecientemente alejados de la identidad española o de las identidades compartidas. Es decir, las Comunidades Autónomas son ahora, como lo eran al principio de la transición, sistemas de articulación territorial plenamente integrados en el Estado pero también en la nación española. Y, en ese sentido, las identidades de los españoles sugieren más bien una base ciudadana de consenso sobre el sistema autonómico diseñado.

Todo lo anterior es especialmente relevante en relación con el caso vasco, puesto que el cuestionamiento principal del consenso sobre el sistema autonómico procede de los partidos nacionalistas vascos. Como señalaba más arriba, ese cuestionamiento se ha ahondado, además, en los últimos años y ha culminado en un proyecto de creación de un Estado libre asociado basado en una ruptura con el Estado a partir de la asunción de la soberanía por parte del País Vasco.

El caso vasco muestra con toda claridad, sin embargo, que no se ha producido un crecimiento del descontento ciudadano o un aumento de las ansias de descentralización, y en este caso de independencia, de los ciudadanos que exigiera una revisión del modelo autonómico y que llevara a la radicalización de los partidos nacionalistas. Al contrario, los datos sobre las actitudes y valores de los vascos en estos años reflejan una gran estabilidad tanto en relación con las actitudes hacia el modelo autonómico como en relación con las identidades. Y, sobre todo, muestran que las posiciones favorables a la independencia no han aumentado en estos años y, por lo tanto, no sostienen la radicalización del nacionalismo vasco y su constante deslegitimación del Estado.

Resulta interesante observar la evolución de los datos relativos a la satisfacción de los vascos con el Estatuto de Autonomía. Porque esos datos nos muestran que los satisfechos y los parcialmente insatisfechos han sumado en los últimos años entre un 60 y un 70 por ciento de los vascos, tal como podemos ver en la tabla 6 . 
TABLA 6

Evolución de la satisfacción de los vascos con el Estatuto de Autonomía

\begin{tabular}{|c|c|c|c|c|c|}
\hline & 1993 & 1998 & 1999 & $2000 / 2$ & $2002 / 6$ \\
\hline (n) & 31 & 43 & 37 & 42 & 30 \\
\hline 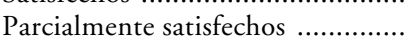 & 25 & 30 & 30 & 19 & 40 \\
\hline 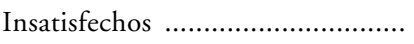 & 26 & 25 & 21 & 27 & 22 \\
\hline$N S / N C \ldots \ldots$ & 18 & 2 & 12 & 12 & 8 \\
\hline
\end{tabular}

Fuente: Euskobarómetro, Universidad del País Vasco, Series temporales.

Otros muchos datos de las series temporales del Euskobarómetro muestran básicamente la misma continuación del rasgo fundamental que estoy destacando en estas páginas, es decir, que incluso en la Comunidad Autónoma donde se produce el más importante cuestionamiento del modelo de articulación territorial del Estado, se observa en estos años una estabilidad en las actitudes de los vascos y una amplia mayoría de las identidades compartidas y de la defensa del modelo autonómico, y no una radicalización de las posiciones que pudiera sostener desde las actitudes y valores de los ciudadanos la radicalización que sí se ha producido en los partidos nacionalistas.

Si observamos los datos relativos a la evolución de las percepciones de identidad de los vascos, llama la atención la notable estabilidad del mantenimiento de las mayoritarias identidades compartidas. Los datos de la última encuesta del Euskobarómetro, de finales del 2002, muestran que el porcentaje de aquellos que se sienten sólo vascos es del 25 por ciento, y ese porcentaje no supone una variación de los datos observados en los últimos veinte años, y se corresponde, por otra parte, con las posiciones independentistas, que se han mantenido también en estos años alrededor de ese porcentaje.

Ahora bien, tanto los datos de la tabla 6 como otros datos de las series temporales del Euskobarómetro como los que se presentan en la tabla 7, relativos a las alternativas sobre la forma de Estado, muestran un cambio llamativo en la última encuesta de 2002. Porque, como podemos ver en la tabla 7 , en la última encuesta del Euskobarómetro de 2002 se observa un importante descenso en el porcentaje de apoyo a la fórmula autonómica y un aumento del apoyo a la fórmula del federalismo. De la misma forma, en la tabla 6 también observábamos un descenso importante del porcentaje de satisfechos con la autonomía. La evaluación de este llamativo dato no es sencilla y, además, requeriría la observación de los resultados de futuras encuestas. Pero, en relación con la tesis mantenida en estas páginas, es decir, la de la responsabilidad de las élites nacionalistas en el cuestionamiento permanente del sistema autonómico, sí cabe destacar que esta variación en el año 2002 se produce después de la radicalización de los partidos nacionalistas o, en otras palabras, 


\section{TABLA 7}

Evolución de las alternativas sobre la forma de Estado en el País Vasco

\begin{tabular}{|c|c|c|c|c|c|}
\hline & 1977 & $1987 / 2$ & 1997 & $2000 / 2$ & $2002 / b$ \\
\hline 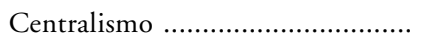 & 9 & 3 & 5 & 9 & 1 \\
\hline 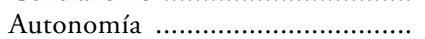 & 29 & 34 & 39 & 44 & 33 \\
\hline 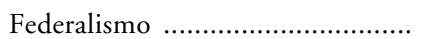 & 32 & 29 & 26 & 22 & 34 \\
\hline Independencia & 24 & 31 & 22 & 26 & 30 \\
\hline NS & 4 & 10 & 7 & 3 & 2 \\
\hline 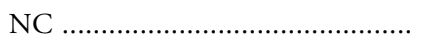 & 2 & 2 & 1 & 2 & - \\
\hline
\end{tabular}

FuENTE: Euskobarómetro, Universidad del País Vasco, Series temporales.

sigue a un cambio de posturas en las posiciones de las élites y partidos nacionalistas.

Los datos sobre las actitudes de los vascos ante los modelos de articulación territorial del Estado y, sobre todo, la evolución de estas actitudes durante todo el periodo democrático indican un predominio de identidades compartidas y de posiciones de aceptación e integración en el marco autonómico desarrollado por el nuevo sistema democrático. No ha existido un problema de insatisfacción ciudadana que explicara la radicalización del nacionalismo vasco.

$\mathrm{Y}$, en términos generales, tampoco ha existido en el conjunto de las Comunidades Autónomas una percepción mayoritaria de crítica al modelo autonómico, de deseos de cambio de ese modelo que puedan explicar la deslegitimación y los debates a los que ha sido sometido. El Estado de las Autonomías es una solución de articulación territorial aceptable y satisfactoria para la gran mayoría de los españoles, y tan sólo se percibe la posibilidad de mejoras técnicas de ese modelo en un porcentaje limitado de ciudadanos.

A partir de esta posición de los ciudadanos, la pregunta que cabe hacerse es la relativa a los factores que explican la posición tendente al disenso y al cuestionamiento del modelo autonómico de las élites políticas nacionalistas. Cabe destacar tres razones que explican esta posición, razones de las cuales citaré brevemente la primera, explicaré la segunda y dedicaré una reflexión algo más pormenorizada a la tercera, en el último apartado de este artículo. El primer factor es el relativo al componente etnicista de los nacionalismos periféricos españoles. Este componente etnicista, que analizo en otro lugar ${ }^{9}$, implica un elemento ideológico profundo de rechazo a lo que se considera otro grupo étnico, el español en este caso, que fundamenta una parte de la incapacidad de estos nacionalismos para integrarse en el consenso alrededor del sistema político español.

9 Véase Edurne Uriarte, España, patriotismo y nación, Espasa Calpe, Madrid, 2003. 
Hay un segundo elemento de especial importancia sobre el que sería interesante profundizar desde la Ciencia Política, y no sólo respecto a los nacionalismos étnicos, sino también respecto a los partidos regionalistas. Me refiero a los intereses de las élites autonómicas y a sus estrategias electorales para asegurarse el mantenimiento del poder autonómico. Esto implica que las élites nacionalistas, o regionalistas, conciben, por un lado, al Gobierno central como un adversario con quien disputan cotas de poder que las élites autonómicas desean aumentar en todo momento. Pero, además, hay una estrategia electoral especialmente útil para esas élites, que es la de la confrontación permanente con el Gobierno central. De esta forma, estas élites, en la medida en que están en el poder en sus respectivas Comunidades, se atribuyen los logros y los éxitos políticos pero achacan los fracasos al Gobierno central. Es decir, consiguen presentarse ante el electorado como Gobierno y oposición al mismo tiempo, y este discurso resulta de enorme utilidad para frenar el lógico desgaste electoral que todo partido afronta tras largos años de permanencia en el poder.

Pero, en tercer lugar, hay otro factor, al que me referiré en el siguiente y último apartado, que es el relativo a las actitudes de los grandes partidos nacionales, Partido Popular y Partido Socialista, ante el modelo autonómico y ante los partidos nacionalistas. El rasgo más significativo de esas actitudes y que explica en parte la prolongación del cuestionamiento del modelo autonómico es el de la falta de consenso suficiente de estos partidos en torno a la cuestión territorial y/o nacionalista en España. Esta ausencia de un consenso suficiente ha facilitado enormemente la capacidad de determinación de los nacionalismos periféricos sobre el conjunto del sistema político español, porque las actitudes y exigencias permanentes de estos nacionalismos no se han encontrado con una respuesta clara y consensuada por parte de los dos grandes partidos nacionales.

\section{LOS PARTIDOS NACIONALES Y EL CONSENSO SOBRE EL ESTADO DE LAS AUTONOMÍAS}

Por factores diversos, los dos grandes partidos nacionales no han llegado en todos estos años a un consenso sólido en torno al modelo de articulación territorial del Estado. Esto no significa que podamos hablar en ninguno de estos partidos de un cuestionamiento del Estado de las Autonomías, pero sí de discursos divergentes en torno a los nacionalismos étnicos, es decir, en torno a los elementos principales de cuestionamiento del modelo autonómico.

Hay factores complejos que explican lo anterior y que están relacionados básicamente con la determinación que el franquismo y la memoria del franquismo han tenido en la cultura política de los españoles y en las actitudes de las élites políticas. La izquierda en general, y el Partido Socialista en particular, compartió la oposición al franquismo junto a los nacionalismos, y, junto a algunos elementos del discurso de la izquierda de los años setenta, esto determinó una cercanía a las posiciones nacionalistas que se ha mantenido después 
durante un prolongado tiempo, a pesar de la evolución de esos nacionalismos en una dirección crecientemente divergente a la del Partido Socialista.

En otro lugar me he referido más detenidamente a esta cuestión ${ }^{10} \mathrm{y}$ he destacado, entre otras cosas, el contexto político de la transición en que se produjo el debate y la definición del modelo de articulación territorial de España. En aquel contexto, y como ha explicado Andrés de Blas, la respuesta al problema regional fue determinada en buena medida por una combinación de los complejos de UCD por el pasado franquista de algunos de sus miembros y las actitudes pro nacionalistas de la izquierda. Andrés de Blas ha señalado, en relación con la derecha y el centro-derecha, que es cierto que el sector más conservador podía echar mano de un difuso regionalismo, herencia de un complejo pasado, ya que el tradicionalismo español fue muy sensible al regionalismo. Pero añade De Blas que todo eso quedó sepultado bajo el alud del franquismo, e influyó escasamente en Alianza Popular y dudosamente en UCD. De Blas señala respecto a UCD que en este caso «debían ser factores ambientales, el peso de la oportunidad política, la desesperada lucha por hacerse con un perfil democrático, los datos claves para entender una actitud ante el problema que en ocasiones desbordaba lo demandado por la pura necesidad de defensa del nuevo sistema democrático»" ${ }^{11}$.

Pero, como también señala Andrés de Blas, algunos comportamientos de la derecha no serían comprensibles, a su vez, sin la actitud de la izquierda. $\mathrm{Y}$, como se ha recordado ya bastantes veces, debemos tener en cuenta que la izquierda, PSOE y PCE, defendía el derecho de autodeterminación y apoyaba las reivindicaciones básicas de los nacionalismos. Las resoluciones sobre las nacionalidades y regiones del Congreso del PSOE de octubre de 1974 defendían el derecho de autodeterminación, del cual se decía que "comporta la facultad de que cada nacionalidad pueda determinar libremente las relaciones que va a mantener con el resto de los pueblos que integran el Estado español», y había un pronunciamiento a favor de la "República federal de las nacionalidades que integran el Estado español $\aleph^{12}$. Y ésa era también la posición del Partido Comunista y del Partido Socialista Popular.

Las anteriores posiciones evolucionaron enormemente en los siguientes años, pero es importante recordarlas porque explican los orígenes y las bases que sustentan el tipo de respuesta dado por los partidos nacionales a la cuestión de la articulación territorial del Estado. En el Partido Socialista las ideas sobre el «derecho de autodeterminación" pronto dejaron de ser asumidas, pero la comprensión hacia las aspiraciones nacionalistas o hacia sus cuestionamientos del Estado de las Autonomías se prolongó hasta el presente. Y los complejos de UCD a los que se refería Andrés de Blas también se han prolongado hasta el presente a través del Partido Popular, cuyo discurso en esta cuestión ha

${ }^{10}$ Edurne Uriarte, España, patriotismo y nación, ob. cit.

11 Andrés de Blas, "Estado de las autonomías y transición política», en Ramón Cotarelo (comp.), Transición política y consolidación democrática (1975-1986), CIS, Madrid, 1992, p. 109.

${ }_{12}$ Andrés de Blas, op. cit., pp. 109 y 110. 
sido muy influido por la preocupación de que pudiera ser relacionado con el franquismo, lo que ha favorecido una cierta indeterminación de ese discurso hasta nuestros días.

Todo ello explica que, todavía hoy en día, el debate sobre el Estado de las Autonomías siga abierto en los partidos políticos y en las élites políticas. La propuesta sobre la alternativa federal que se hace desde la izquierda en general, y muy especialmente desde algunos sectores del Partido Socialista, es el exponente quizá más llamativo de este debate. El federalismo es un concepto central del discurso del Partido Socialista sobre la articulación del Estado y, junto al Partido Socialista, también de una parte de los intelectuales. Cuando el PSOE expone sus alternativas, suele aludir al federalismo.

La influencia del concepto de federalismo es tan grande que también muchas encuestas politológicas, como el Euskobarómetro citado más arriba, incluyen al federalismo como una alternativa al modelo autonómico. Y es aquí donde se introduce una enorme confusión en el debate político español y se entienden, además, las dudas que existen en España en torno al significado del modelo autonómico.

Porque el modelo autonómico es ya un modelo federal, y el planteamiento de que existiría una supuesta alternativa federal a ese modelo indica una parte de los problemas que todavía siguen abiertos en nuestro país en esta materia. Como han señalado numerosos autores, la diferencia entre un Estado unitario y un Estado federal es que en el segundo hay un doble nivel de poder, es decir, hay ámbitos de actuación y de decisión propios de la unidad local o descentralizada y ámbitos propios de la unidad mayor. Hay una definición de Robert Dahl, citada por Juan Linz, que es interesante recordar: "Por federalismo entendemos aquel sistema en el que algunos asuntos están dentro de la competencia exclusiva de ciertas unidades locales — cantones, estados y provinciasy están constitucionalmente más allá del alcance de la autoridad del gobierno nacional, y donde otros asuntos están constitucionalmente fuera del alcance de la autoridad de las unidades menores» ${ }^{13}$.

Pero, como ha dicho Juan Linz, no todos los Estados que cumplen la condición de federales se llaman a sí mismos federales. Algunos, continuaba Juan Linz, se han dado otros nombres, como es el caso de España y el concepto de Estado de las Autonomías ${ }^{14}$. O, como ha señalado Eliseo Aja, «(...) el Estado autonómico comparte los caracteres esenciales de los estados federales, y aunque tal afirmación pueda sorprender porque la Constitución no utiliza este término, es evidente que la estructura de poder en España corresponde a la propia de los estados federales actuales» $»^{15}$.

Pero, para clarificar aún más la confusión todavía existente en España entre

13 Juan Linz, "Democracia, multinacionalismo y federalismo», Revista Española de Ciencia Politica, n. ${ }^{\circ} 1$, octubre 1999 , p. 8.

14 Juan Linz, op. cit., pp. 8 y 9.

15 Eliseo Aja, El Estado autonómico. Federalismo y hechos diferenciales, Alianza, Madrid, 1999, pp. 80 y 81 . 
el concepto de federalismo y el de autonomía, conviene recordar algunas otras puntualizaciones de estos dos autores. Eliseo Aja ha señalado que es importante desmentir la idea, muy generalizada, de que sólo existe federación cuando nace de la fusión de varios Estados previamente independientes, y no cuando el Estado unitario se descentraliza o federaliza. Porque casos como los de Austria, Canadá, Australia o Bélgica demuestran que esto no es así. También señala Aja que hay que tener en cuenta que el Estado federal no presenta un modelo único y que encontramos importantes diferencias en instituciones, en competencias o en las relaciones entre la federación y los Estados, en los distintos Estados federales existentes. Y Aja añade también otro dato muy importante, y es que ningún Estado federal reconoce la soberanía de sus Estados miembros ${ }^{16}$.

A todas estas precisiones de Aliseo Aja habría que añadir una reflexión muy importante de Juan Linz en relación con la existencia de una segunda cámara. Señala Linz que, en su opinión, la existencia de una segunda cámara no es una condición necesaria para un Estado federal dado que el ámbito de poder constitucionalmente reservado al demos de la unidad se puede garantizar sin esa segunda cámara. Y, además, continúa Linz, incluir ese criterio obligaría a excluir a una serie de Estados que son federales ${ }^{17}$.

Y, por último, y para completar esta aclaración conceptual, es también importante añadir otra precisión que Juan Linz considera la distinción más importante entre los Estados federales. Se trata de la distinción entre Estados federales de nacionalidad única y Estados federales multinacionales. Suiza, por ejemplo, a pesar de su carácter multilingüístico, multicultural y multirreligioso, es para los ciudadanos suizos una nación-Estado. Linz también aporta el ejemplo de Austria, que después de 1918 era un Estado en el que muchos ciudadanos se sentían parte de la nación alemana y cuestionaban la legitimidad del Estado que se les había impuesto. Tras la II Guerra Mundial se convirtió para muchos austriacos en una nación-Estado pero que no se sentía como una nación separada, y sólo lentamente ha adquirido una conciencia de Estadonación.

Y añade Linz que, por otro lado, tenemos sociedades multinacionales, multilingües y multiculturales en las que para la mayoría de los ciudadanos hay una identificación nacional común y para muchos otros la conciencia de ser una nación-Estado: «Es, por lo tanto, posible hablar de algo como un continuum en el que el Estado federal mononacional esté en un extremo y el Estado federal multinacional, basado en débiles vínculos emocionales con el Estado, esté en el otro extremo»" ${ }^{18}$. Juan Linz no añade en qué punto exacto de ese continuum podríamos situar a España, pero es evidente, en primer lugar, que es un Estado federal multinacional y, en segundo lugar, que en algunas de sus unidades existen problemas de vínculos emocionales débiles con el Estado.

${ }^{16}$ Eliseo Aja, op. cit., pp. 27-31.

${ }_{17}$ Juan Linz, op. cit., p. 10.

18 Juan Linz, op. cit., p. 17. 
En definitiva, en España tenemos un Estado federal, pero, sin embargo, todavía se plantea el modelo federal como una alternativa al Estado autonómico. Y esto nos da una idea de la debilidad o de las confusiones o vacilaciones en los partidos españoles frente a ese modelo autonómico. El planteamiento del federalismo como una alternativa al actual modelo refleja fundamentalmente una continuación de algunas de las claves del discurso de la izquierda del inicio de la transición, y básicamente la búsqueda permanente de respuestas a las demandas de los nacionalismos periféricos.

La memoria del franquismo también ha determinado hasta muy recientemente el discurso del Partido Popular en torno al modelo autonómico y las demandas nacionalistas. En este caso, con una cierta falta de interés por abordar una problemática que pudiera hacer surgir acusaciones de recuerdo del franquismo. Y ha contribuido de esta forma a alimentar la capacidad de determinación de los nacionalismos étnicos sobre el debate en torno al Estado de las Autonomías.

El surgimiento del concepto de "patriotismo constitucional» muestra una renovación del discurso de los partidos nacionales sobre el modelo autonómico y sobre la articulación del Estado con la nación española, por un lado, y con las distintas identidades regionales o nacionales, por otro lado. La interrogante que se plantea es en qué medida este concepto ha sido asumido únicamente por el Partido Popular o tiene capacidad para trascender la división izquier$\mathrm{da} /$ derecha y supone, por lo tanto, el inicio de un cambio significativo en las relaciones de los dos principales partidos con la definición del significado y de los objetivos futuros del Estado de las Autonomías.

\section{ABSTRACT}

This article analyses what is considered to be the unsolved problem of Spanish democracy, which is consensus regarding the territorial articulation of the State. It defends the view that the lack of satisfaction and debate that have arisen regarding the State of Autonomous Communities do not necessarily come from society or from unsatisfied citizens, but from the attitudes of political parties and élites. Information gathered from the opinions held by Spaniards, even in the most "problematical» of autonomous regional communities, show a high or significant degree of satisfaction with the autonomous regional community system. This article also puts forward the argument that dissatisfaction is essentially explainable because for the autonomous regional élites, it is an effective electoral asset and an instrument for perpetuating the huge political and social power attained in their territories. Added to this main factor is the lack of sufficient consensus from the two main Spanish parties, PP (conservative) and PSOE (socialist), regarding the model for territorial articulation in Spain. 